\section{Closed-Form Green's Function Representations for Mutual Coupling Calculations Between Apertures on a Perfect Electric Conductor Circular Cylinder Covered With Dielectric Layers}

\author{
M. S. Akyüz, V. B. Ertürk, and M. Kalfa
}

\begin{abstract}
Closed-form Green's function (CFGF) representations are developed for tangential magnetic current sources to calculate the mutual coupling between apertures on perfectly conducting circular cylinders covered with dielectric layers. The new representations are obtained by first rewriting the corresponding spectral domain Green's function representations in a different form (so that accurate results for electrically large cylinders, and along the axial line of a cylinder can be obtained). Then, the summation over the cylindrical eigenmodes is calculated efficiently. Finally, the resulting expressions are transformed to the spatial domain using a modified two-level generalized pencil of function method. Numerical results are presented showing good agreement when compared to CST Microwave Studio results.
\end{abstract}

Index Terms-Aperture antennas, closed-form Green's functions, generalized pencil of function (GPOF) method, mutual coupling.

\section{INTRODUCTION}

A wide range of military and commercial applications require accurate and efficient analysis tools to investigate waveguide-fed aperture antennas/arrays on perfect electric conductors (PEC) covered by dielectric layer(s) ([1], [2]). A number of integral equation (IE) based tools have been developed in the past that use closed-form Green's function (CFGF) representations as the kernel of the IE [3]-[5]. However, similar tools are not available for cylindrical structures, because the available CFGF representations ([6]-[11]) are not valid when the problem of interest is the mutual coupling between two apertures on a PEC cylinder covered by dielectric layer(s). Recently, novel CFGF representations that are accurate for almost all possible source and field points are presented in [12] to investigate microstrip antennas/arrays on cylindrically stratified media. However, there is no counterpart of [12] for aperture type antennas in the literature. Therefore, in this communication, we provide CFGF representations for magnetic sources to investigate waveguide-fed aperture antennas/arrays on PEC cylinders covered by one or more than one dielectric layers.

Our approach starts by modeling an aperture antenna on the PEC surface with a tangential magnetic current mode by invoking the surface equivalence theorem [13]. Then, the methodology presented in [12] is followed. Briefly, the conventional spectral domain Green's function representations ([6], [7]) of a tangential magnetic type current source is rewritten in such a way that all the special cylindrical functions (such as Bessel and Hankel functions along with their derivatives) are represented in the form of ratios. Thus, possible numerical problems in

Manuscript received June 25, 2010; revised November 12, 2010; accepted January 15, 2011. Date of publication June 07,2011 ; date of current version August 03, 2011. This work was supported in part by the Turkish Scientific and Technological Research Council (TÜBİTAK) under grant no EEEAG-104E044 and in part by the Turkish Academy of Sciences (TÜBA)-GEBIP.

M. S. Akyüz and M. Kalfa are with the Department of Electrical and Electronics Engineering, Bilkent University, TR-06800 Bilkent, Ankara, Turkey and also with Aselsan Electronics Inc., Ankara, Turkey.

V. B. Ertürk is with the Department of Electrical and Electronics Engineering, Bilkent University, TR-06800 Bilkent, Ankara, Turkey (e-mail: vakur@ee.bilkent.edu.tr).

Color versions of one or more of the figures in this communication are available online at http://ieeexplore.ieee.org.

Digital Object Identifier 10.1109/TAP.2011.2158787 the computations of these functions are avoided. Because the spectral domain representations of the cylindrical Green's function contains a summation over the cylindrical eigenmodes $n$, and a Fourier integral over $k_{z}$, available acceleration techniques that have been used in [12] are implemented to perform the summation efficiently and to handle numerical problems along the integration path. Finally, the resulting expressions are transformed to the spatial domain by taking the $k_{z}$ integration in closed-form with the help of the generalized pencil of function method (GPOF) [14].

Note that similar to that of [12], the proposed CFGF representations (for magnetic sources) in this communication are valid in a significantly wider source-field point region compared to previously available CFGF representations, and can be used in the investigation of mutual coupling between two aperture antennas (in addition to radiation/scattering problems where $\rho \neq \rho^{\prime}$ ). Such region includes the axial line of the cylinder (i.e., $\rho=\rho^{\prime}$ and $\phi=\phi^{\prime}$ ). Because of several techniques used in the course of CFGF derivations to improve the efficiency and accuracy, the proposed CFGF representations are accurate both for electrically small and relatively large cylinders, where the latter case has been usually accomplished using high-frequency based techniques ([1], [2]). However, the summation over $n$ may exhibit some convergence problems for electrically very large cylinders. Besides, the proposed CFGF expressions are not valid in the source region where two magnetic current modes touch/overlap with each other.

In Section II, the geometry and the derivation of the CFGF representations are presented. The expressions are written in the same form of those presented in [12] so that similar methodology can be followed. Numerical results in the form of mutual coupling between two waveguide-fed aperture antennas on a PEC cylinder covered with a dielectric layer are given in Section III to assess the accuracy of the method. An $e^{j \omega t}$ time dependence, with $\omega$ being the angular frequency, is assumed and suppressed throughout this communication. Note also that $G_{u v}$ stands for the $u v$ component for the spatial domain Green's function of a magnetic field due to a magnetic current mode, whereas $\tilde{G}_{u v}$ is its spectral domain counterpart.

\section{FORMULATION}

Fig. 1 illustrates the geometry of two identical apertures on an infinitely long PEC cylinder (along the $z$-axis) covered with a dielectric layer and the outermost region is free-space. The PEC cylinder has a radius of $a_{0}$, the dielectric layer has a thickness of $t_{h}$ (hence, $a_{1}=a_{0}+t_{h}$ ), permittivity of $\epsilon_{1}=\epsilon_{0} \epsilon_{r}$ and permeability $\mu_{0}$. The dimension of each aperture is $z_{a} \times l_{a}\left(l_{a}=a_{0} \Delta \phi\right)$ in the $z$ - and $l$-directions, respectively, with $l=a_{0} \phi$. In Fig. $1, s$ denotes the geodesic path distance between the apertures and $\alpha$ is the angle between the geodesic path and the $l$-axis.

Similar to [12], the spectral domain Green's function components for tangential magnetic sources $\tilde{G}_{u v}(u=z$ or $\phi, v=z$ or $\phi)$, originally given in [6], [7] for $\rho \neq \rho^{\prime}$, are rewritten for $\rho=\rho^{\prime}$ as

$$
\begin{aligned}
\frac{\tilde{G}_{u v}}{k_{z}^{m}}= & -\frac{1}{4 \omega} \sum_{n=-\infty}^{\infty}\left(k_{\rho_{j}}^{2}\right)^{q} n^{p} H_{n}^{(2)}\left(k_{\rho_{j}} \rho\right) J_{n}\left(k_{\rho_{j}} \rho^{\prime}\right) \\
& \times f_{u v}\left(n, k_{z}\right) e^{j n}(\Delta \phi)
\end{aligned}
$$

where $\Delta \phi=\phi-\phi^{\prime}$, and for $u v=z z: p=0, q=1, m=0$, for $u v=\phi z=z \phi: p=1, q=0, m=1$, and for $u v=\phi \phi: p=2$, $q=0, m=0$. Note that (1) is exactly in the same generic form as that of an electric current mode ([12]). This is very advantageous because from now on, all the steps and techniques that have been presented in [12] to improve the efficiency and accuracy can be implemented in 


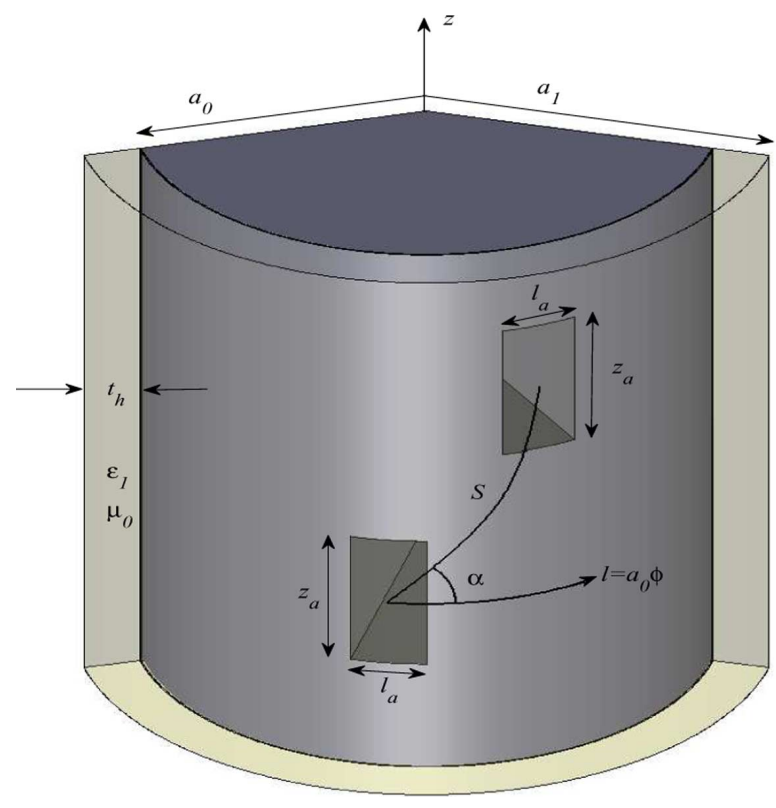

Fig. 1. Geometry of the problem.

the same fashion. However, the term $f_{u v}\left(n, k_{z}\right)$ differs from those of electric current mode for each component and is explicitly given by

$$
\begin{aligned}
f_{z z}\left(n, k_{z}\right)= & \frac{\overline{\mathbf{F}}_{r 1}(2,2)}{\mu_{j}} \\
f_{\phi z}\left(n, k_{z}\right)= & \frac{1}{\mu_{j} \rho} \overline{\mathbf{F}}_{r 1}(2,2)-\frac{j \omega \epsilon_{j} k_{\rho_{j}}}{\mu_{j} k_{z}} \overline{\mathbf{F}}_{r 2}(1,2) \\
f_{z \phi}\left(n, k_{z}\right)= & \frac{1}{\mu_{j} \rho^{\prime}} \overline{\mathbf{F}}_{r 1}(2,2)+\frac{j \omega k_{\rho_{j}}}{k_{z}} \overline{\mathbf{F}}_{r 3}(2,1) \\
f_{\phi \phi}\left(n, k_{z}\right)= & \frac{k_{z}^{2}}{\mu_{j} \rho \rho^{\prime} k_{\rho_{j}}^{2}} \overline{\mathbf{F}}_{r 1}(2,2)-\frac{j \omega \epsilon_{j} k_{z}}{k_{\rho_{j}} \mu_{j} \rho^{\prime}} \overline{\mathbf{F}}_{r 2}(1,2) \\
& +\frac{j \omega n k_{z}}{k_{\rho_{j}} \rho} \overline{\mathbf{F}}_{r 3}(2,1) \\
& +\frac{H_{n}^{\prime(2)}\left(k_{\rho_{j}} \rho\right)}{n H_{n}^{(2)}\left(k_{\rho_{j}} \rho\right)} \frac{J_{n}^{\prime}\left(k_{\rho_{j}} \rho^{\prime}\right)}{n J_{n}\left(k_{\rho_{j}} \rho^{\prime}\right)} \omega^{2} \epsilon_{j} \overline{\mathbf{F}}_{r 4}(1,1)
\end{aligned}
$$

where $\overline{\mathbf{F}}_{r k}(i, j)$ with $k=1, \ldots, 4$ is the $(i, j)$ th entry $(1 \leq i, j \leq 2)$ of the $2 \times 2$ matrix $\overline{\mathbf{F}}_{r k}$ expressed as

$$
\begin{aligned}
& \overline{\mathbf{F}}_{r 1}=\left[\overline{\mathbf{I}}+\frac{H_{n}^{(2)}\left(k_{\rho_{j}} a_{j}\right)}{H_{n}^{(2)}\left(k_{\rho_{j}} \rho\right)} \frac{J_{n}\left(k_{\rho_{j}} \rho\right)}{J_{n}\left(k_{\rho_{j}} a_{j}\right)} \tilde{\overline{\mathbf{R}}} r_{j, j+1}\right] \tilde{\mathbf{\mathbf { M }}_{j+}} \\
& \times\left[\overline{\mathbf{I}}+\frac{J_{n}\left(k_{\rho_{j}} a_{j-1}\right)}{J_{n}\left(k_{\rho_{j}} \rho^{\prime}\right)} \frac{H_{n}^{(2)}\left(k_{\rho_{j}} \rho^{\prime}\right)}{H_{n}^{(2)}\left(k_{\rho_{j}} a_{j-1}\right)} \tilde{\mathbf{\mathbf { R }}} r_{j, j-1}\right] \\
& \overline{\mathbf{F}}_{r 2}=\left[\frac{H_{n}^{\prime(2)}\left(k_{\rho_{j}} \rho\right)}{n H_{n}^{(2)}\left(k_{\rho_{j}} \rho\right)} \overline{\mathbf{I}}+\frac{H_{n}^{(2)}\left(k_{\rho_{j}} a_{j}\right)}{H_{n}^{(2)}\left(k_{\rho_{j}} \rho\right)} \frac{J_{n}^{\prime}\left(k_{\rho_{j}} \rho\right)}{n J_{n}\left(k_{\rho_{j}} a_{j}\right)} \tilde{\overline{\mathbf{R}}} r_{j, j+1}\right] \\
& \times \tilde{\mathbf{M}}_{j+}\left[\overline{\mathbf{I}}+\frac{J_{n}\left(k_{\rho_{j}} a_{j-1}\right)}{J_{n}\left(k_{\rho_{j}} \rho^{\prime}\right)} \frac{H_{n}^{(2)}\left(k_{\rho_{j}} \rho^{\prime}\right)}{H_{n}^{(2)}\left(k_{\rho_{j}} a_{j-1}\right)} \tilde{\mathbf{\mathbf { R }}} r_{j, j-1}\right] \\
& \overline{\mathbf{F}}_{r 3}=\left[\overline{\mathbf{I}}+\frac{J_{n}\left(k_{\rho_{j}} \rho\right)}{J_{n}\left(k_{\rho_{j}} a_{j}\right)} \frac{H_{n}^{(2)}\left(k_{\rho_{j}} a_{j}\right)}{H_{n}^{(2)}\left(k_{\rho_{j}} \rho\right)} \tilde{\mathbf{\mathbf { R }}} r_{j, j+1}\right] \tilde{\overline{\mathbf{M}}_{j+}} \\
& \times\left[\frac{J_{n}^{\prime}\left(k_{\rho_{j}} \rho^{\prime}\right)}{n J_{n}\left(k_{\rho_{j}} \rho^{\prime}\right)} \overline{\mathbf{I}}\right. \\
& \left.+\frac{J_{n}\left(k_{\rho_{j}} a_{j-1}\right)}{J_{n}\left(k_{\rho_{j}} \rho^{\prime}\right)} \frac{H_{n}^{\prime(2)}\left(k_{\rho_{j}} \rho^{\prime}\right)}{n H_{n}^{(2)}\left(k_{\rho_{j}} a_{j-1}\right)} \tilde{\overline{\mathbf{R}}} r_{j, j-1}\right]
\end{aligned}
$$

$$
\begin{aligned}
\overline{\mathbf{F}}_{r 4}= & {\left[\overline{\mathbf{I}}+\frac{H_{n}^{(2)}\left(k_{\rho_{j}} a_{j}\right)}{H_{n}^{\prime(2)}\left(k_{\rho_{j}} \rho\right)} \frac{J_{n}^{\prime}\left(k_{\rho_{j}} \rho\right)}{J_{n}\left(k_{\rho_{j}} a_{j}\right)} \tilde{\mathbf{\mathbf { R }}} r_{j, j+1}\right] \tilde{\mathbf{M}}_{j+} } \\
& \times\left[\overline{\mathbf{I}}+\frac{J_{n}\left(k_{\rho_{j}} a_{j-1}\right)}{J_{n}^{\prime}\left(k_{\rho_{j}} \rho^{\prime}\right)} \frac{H_{n}^{\prime(2)}\left(k_{\rho_{j}} \rho^{\prime}\right)}{H_{n}^{(2)}\left(k_{\rho_{j}} a_{j-1}\right)} \tilde{\overline{\mathbf{R}}} r_{j, j-1}\right]
\end{aligned}
$$

$\overline{\mathbf{I}}$ in (6)-(9) is the $2 \times 2$ identity matrix, and the expression $\tilde{\mathbf{M}}_{j+}$ in (6)-(9) contains the $2 \times 2$ generalized reflection and transmission matrices $\tilde{\overline{\mathbf{R}}}$ and $\tilde{\mathbf{T}}$, respectively, all of which are explicitly given in [6]. Finally in (1)-(9), ' denotes the derivative with respect to the argument, and $k_{\rho_{j}}=\sqrt{k_{j}^{2}-k_{z}^{2}}$ with $k_{j}$ being the wave number of the medium $\left(k_{j}=\sqrt{\epsilon_{r_{j}}} k_{0}\right)$.

Note that in (1), all Bessel and Hankel functions are in the form of ratios. Besides, Debye representations [15] of the ratios are found in closed-form and used during the summation over $n$ when necessary. Thus, efficiency is improved and possible numerical problems in the computations of these functions are avoided, in particular for large $n$ values. Moreover, by applying an envelope extraction method with respect to $n$ to (1), the efficiency and accuracy of the summation are further improved. Thus, (1) becomes

$$
\begin{aligned}
\frac{\tilde{G}_{u v}}{k_{z}^{m}}=-\frac{1}{4 \omega} & \left\{\sum_{n=-\infty}^{\infty}\left(k_{\rho_{j}}^{2}\right)^{q} n^{p} H_{n}^{(2)}\left(k_{\rho_{j}} \rho\right) J_{n}\left(k_{\rho_{j}} \rho^{\prime}\right)\right. \\
& \times\left[f_{u v}\left(n, k_{z}\right)-C_{u v}\left(k_{z}\right)\right] e^{j n \Delta \phi} \\
& \left.+C_{u v}\left(k_{z}\right)\left(k_{\rho_{j}}^{2}\right)^{q} F_{1}^{u v}\left[H_{0}^{(2)}\left(k_{\rho_{j}}\left|\bar{\rho}-\bar{\rho}^{\prime}\right|\right)\right]\right\} .
\end{aligned}
$$

In (10), $C_{u v}\left(k_{z}\right)$ is the limiting value of $f_{u v}\left(n, k_{z}\right)$ for very large $n$ values. It is obtained numerically and is constant with respect to $n$. Also in (10), $F_{1}^{u v}[\cdot]$ is a function of $H_{0}^{(2)}\left(k_{\rho_{j}}\left|\bar{\rho}-\bar{\rho}^{\prime}\right|\right)$ and is given for each component as

$$
\begin{aligned}
F_{1}^{z z}\left[H_{0}^{(2)}\left(k_{\rho_{j}}\left|\bar{\rho}-\bar{\rho}^{\prime}\right|\right)\right] & =H_{0}^{(2)}\left(k_{\rho_{j}}\left|\bar{\rho}-\bar{\rho}^{\prime}\right|\right) \\
F_{1}^{\phi z}\left[H_{0}^{(2)}\left(k_{\rho_{j}}\left|\bar{\rho}-\bar{\rho}^{\prime}\right|\right)\right] & =F_{1}^{z \phi}\left[H_{0}^{(2)}\left(k_{\rho_{j}}\left|\bar{\rho}-\bar{\rho}^{\prime}\right|\right)\right] \\
& =-j \frac{\partial H_{0}^{(2)}\left(k_{\rho_{j}}\left|\bar{\rho}-\bar{\rho}^{\prime}\right|\right)}{\partial \phi} \\
F_{1}^{\phi \phi}\left[H_{0}^{(2)}\left(k_{\rho_{j}}\left|\bar{\rho}-\bar{\rho}^{\prime}\right|\right)\right] & =\frac{\partial^{2} H_{0}^{(2)}\left(k_{\rho_{j}}\left|\bar{\rho}-\bar{\rho}^{\prime}\right|\right)}{\partial \phi \partial \phi^{\prime}}
\end{aligned}
$$

The addition theorem of Hankel function ([15]) is used in the course of obtaining (11)-(13). Explicit expressions for (12) and (13) are in [12].

As the next step, first the integration path is deformed as explained in [12] to be away from the problems related to branch-point and pole singularities. Then, another envelope extraction with respect to $k_{z}$ is applied to (10), to avoid numerical problems due to the imaginary part of (10) that appears when $k_{z}$ is large and $\Delta \phi$ is small. As a result, the spatial domain Green's function expression becomes

$$
\begin{aligned}
G_{u v}= & \left(\frac{j \partial}{\partial z}\right)^{m}\left\{\frac { 1 } { 2 \pi } \int _ { - \infty } ^ { \infty } \left(\frac{\tilde{G}_{u v}}{k_{z}^{m}}+\frac{1}{4 \omega} C_{u v}\left(k_{z \infty}\right)\left(k_{\rho_{j}}^{2}\right)^{q}\right.\right. \\
F_{1}^{u v} & {\left.\left[H_{0}^{(2)}\left(k_{\rho_{j}}\left|\bar{\rho}-\bar{\rho}^{\prime}\right|\right)\right]\right) e^{-j k_{z}\left(z-z^{\prime}\right)} d k_{z} } \\
& \left.-\frac{j}{4 \pi \omega} C_{u v}\left(k_{z \infty}\right)\left(k_{j}^{2}+\frac{\partial^{2}}{\partial z^{2}}\right)^{q} F_{2}^{u v}\left[I_{1}\right]\right\}
\end{aligned}
$$

where

$$
F_{2}^{z z}\left[I_{1}\right]=\frac{e^{-j k_{j}\left|\bar{r}-\bar{r}^{\prime}\right|}}{\left|\bar{r}-\bar{r}^{\prime}\right|}
$$




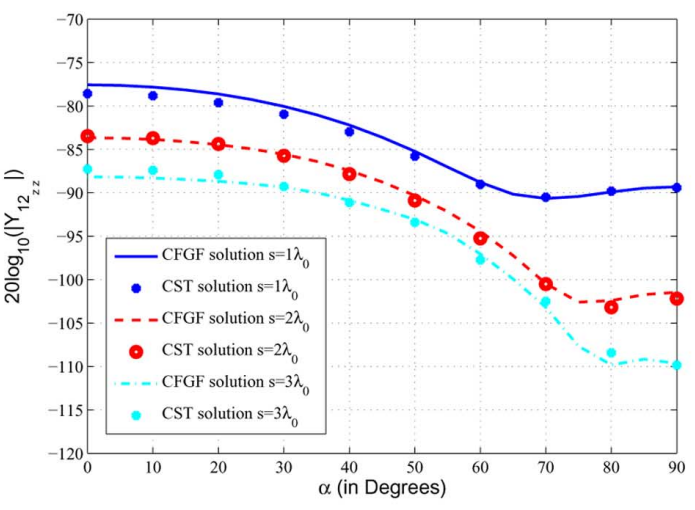

(a)

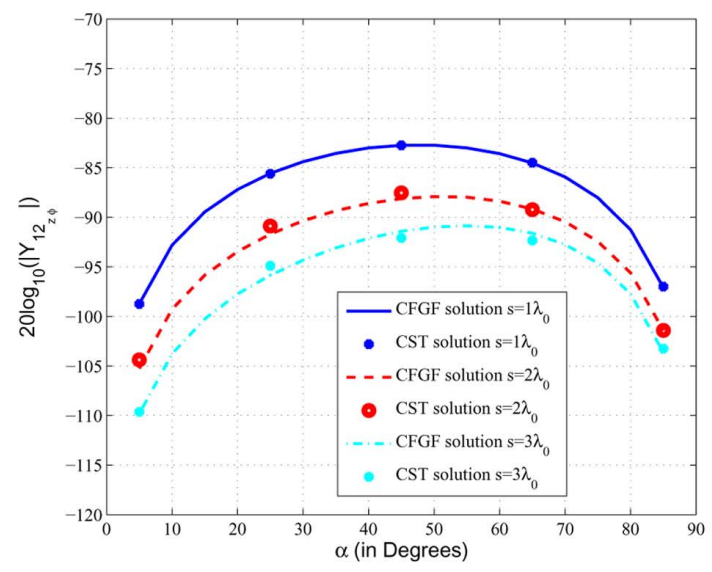

(c)

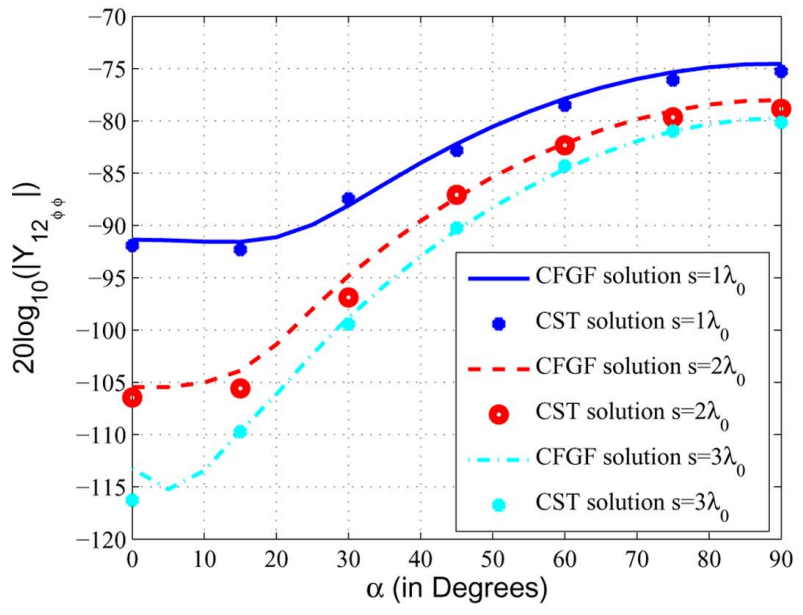

(e)

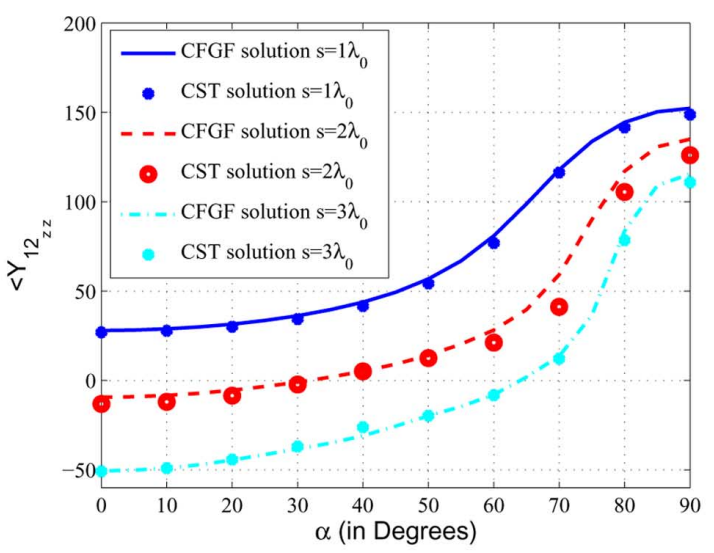

(b)

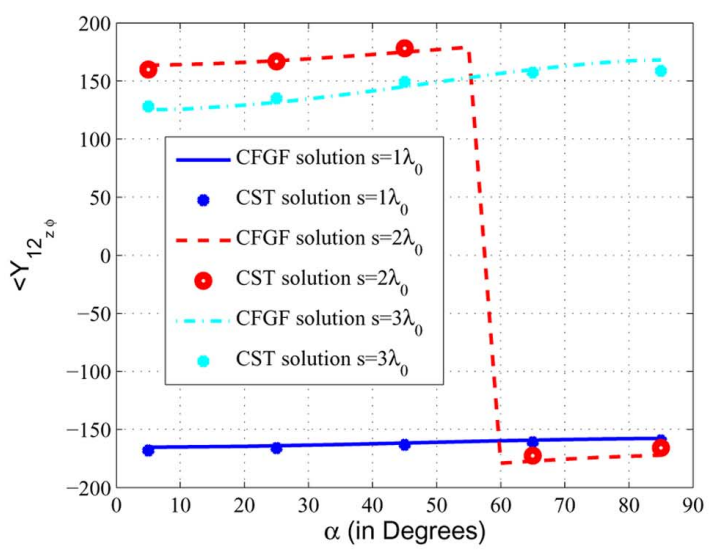

(d)

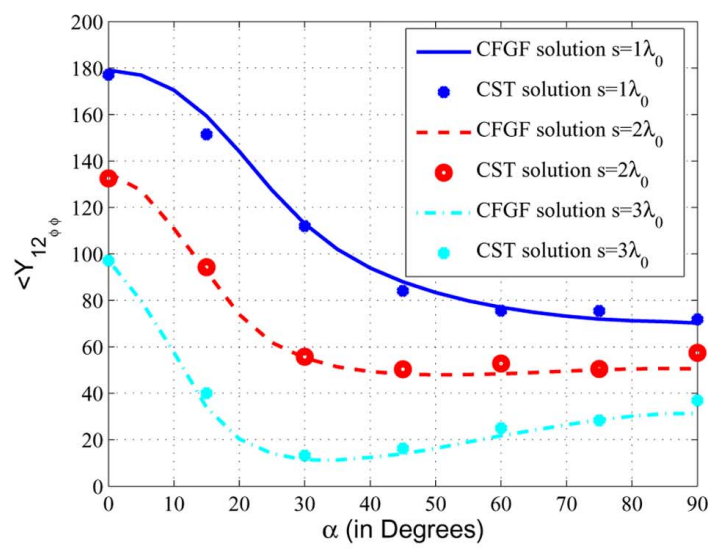

(f)

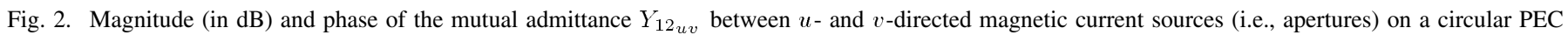
cylinder coated with a dielectric layer. The cylinder parameters are $a_{0}=3 \lambda_{0}, a_{1}=3.06 \lambda_{0}, \epsilon_{r}=3.25$, and the aperture parameters are $0.3 \lambda_{0}$ by $0.1 \lambda_{0}$.

$$
\begin{aligned}
F_{2}^{\phi z}\left[I_{1}\right]= & F_{2}^{z \phi}\left[I_{1}\right] \\
= & -j \frac{\rho^{\prime} \rho}{\left|\bar{\rho}-\bar{\rho}^{\prime}\right|} \sin \left(\phi-\phi^{\prime}\right) \frac{\partial I_{1}}{\partial\left|\bar{\rho}-\bar{\rho}^{\prime}\right|} \\
F_{2}^{\phi \phi}\left[I_{1}\right]= & {\left[-\frac{\rho^{\prime} \rho}{\left|\bar{\rho}-\bar{\rho}^{\prime}\right|} \cos \left(\phi-\phi^{\prime}\right)\right.} \\
& \left.+\frac{\rho^{\prime 2} \rho^{2}}{\left|\bar{\rho}-\bar{\rho}^{\prime}\right|^{3}} \sin ^{2}\left(\phi-\phi^{\prime}\right)\right] \frac{\partial I_{1}}{\partial\left|\bar{\rho}-\bar{\rho}^{\prime}\right|} \\
& -\frac{\rho^{\prime 2} \rho^{2}}{\left|\bar{\rho}-\bar{\rho}^{\prime}\right|^{2}} \sin ^{2}\left(\phi-\phi^{\prime}\right) \frac{\partial^{2} I_{1}}{\partial\left|\bar{\rho}-\bar{\rho}^{\prime}\right|^{2}}
\end{aligned}
$$

and $C_{u v}\left(k_{z \infty}\right)$ is the value of $C_{u v}\left(k_{z}\right)$ on the last path of the deformed integration path for asymptotically large $k_{z}$ values. Note that in arriving at (15)-(17) the following relations are used:

$$
\begin{aligned}
I_{1}=\frac{e^{-j k_{j}\left|\bar{r}-\bar{r}^{\prime}\right|}}{\left|\bar{r}-\bar{r}^{\prime}\right|} & =\frac{-j}{2} \int_{-\infty}^{\infty} H_{0}^{(2)}\left(k_{\rho_{j}}\left|\bar{\rho}-\bar{\rho}^{\prime}\right|\right) e^{-j k_{z}\left(z-z^{\prime}\right)} d k_{z} \\
\frac{\partial I_{1}}{\partial\left|\bar{\rho}-\bar{\rho}^{\prime}\right|} & =\frac{-j}{2} \int_{-\infty}^{\infty} k_{\rho_{j}} H_{0}^{\prime(2)}\left(k_{\rho_{j}}\left|\bar{\rho}-\bar{\rho}^{\prime}\right|\right) e^{-j k_{z}\left(z-z^{\prime}\right)} d k_{z}
\end{aligned}
$$




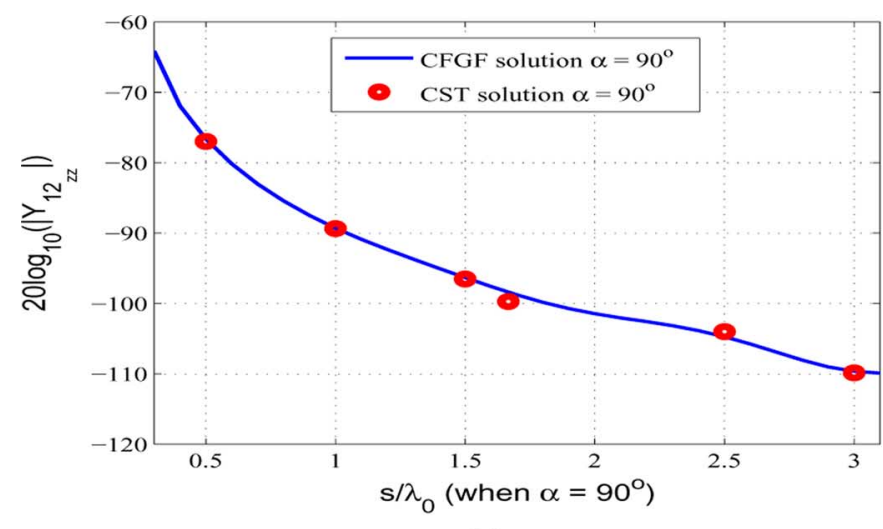

(a)

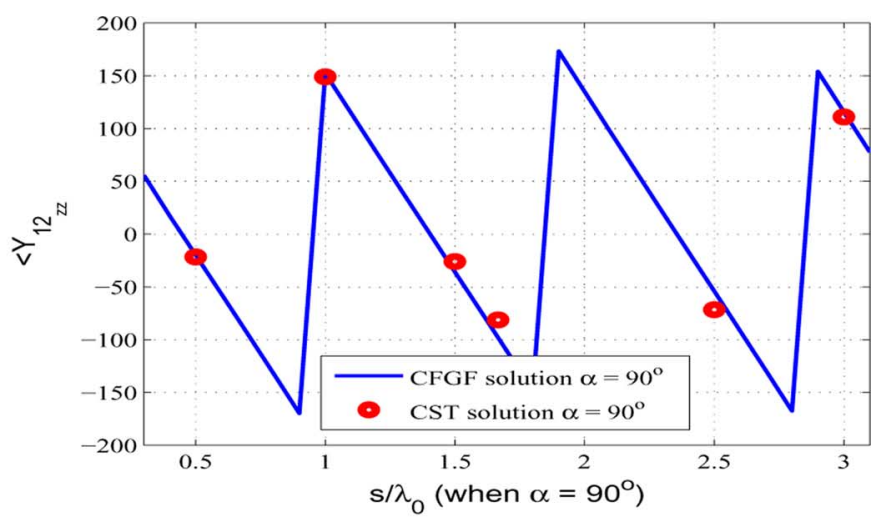

(b)

Fig. 3. Magnitude (in $\mathrm{dB}$ ) and phase of the mutual admittance $Y_{12 z z}$ between two identical $z$-directed current sources versus separation when $\alpha=90^{\circ}$ (axial line) for the coated cylinder with the parameters given in Fig. 2.

$$
\frac{\partial^{2} I_{1}}{\partial\left|\bar{\rho}-\bar{\rho}^{\prime}\right|^{2}}=\frac{-j}{2} \int_{-\infty}^{\infty} k_{\rho_{j}}^{2} H_{0}^{\prime \prime(2)}\left(k_{\rho_{j}}\left|\bar{\rho}-\bar{\rho}^{\prime}\right|\right) e^{-j k_{z}\left(z-z^{\prime}\right)} d k_{z} .
$$

Note also that, the integral part of (14) is well-behaved (except along the axial line) and hence, GPOF is applied to this part to obtain CFGF expressions whereas the second term of (14) is already in closed-form.

Before the implementation of GPOF, the last step is to resolve the axial line $\left(\rho=\rho^{\prime}\right.$ and $\left.\phi=\phi^{\prime}\right)$ problem which is performed exactly the same way as explained in [12]. Briefly, the $H_{0}^{(2)}\left(k_{\rho_{j}}\left|\bar{\rho}-\bar{\rho}^{\prime}\right|\right)$ related terms in (14) exhibits a logarithmic singularity (which is integrable) along the axial line. The small argument approximation of $H_{0}^{(2)}\left(k_{\rho_{j}}\left|\bar{\rho}-\bar{\rho}^{\prime}\right|\right)$ along the axial line yields

$$
H_{0}^{(2)}\left(k_{\rho_{j}}\left|\bar{\rho}-\bar{\rho}^{\prime}\right|\right) \approx 1-j \frac{2}{\pi} \log \left(\gamma k_{\rho_{j}} \rho\right)-j \frac{2}{\pi} \log \left(\frac{\Delta \phi}{2}\right)
$$

where $\gamma=1.781$. The last term, which is the logarithmic singularity part, is isolated. The contribution coming from its integration over the surface areas of basis and testing magnetic current modes during a mutual admittance calculation is exactly zero. On the other hand, for the terms that involve the derivatives of $H_{0}^{(2)}\left(k_{\rho_{j}}\left|\bar{\rho}-\bar{\rho}^{\prime}\right|\right)$ with respect to $\phi$ and $\phi^{\prime}$, an integration by parts with respect to $\phi$ and $\phi^{\prime}$ is performed first. This step obviously requires the condition that the selected basis and testing current modes should be differentiable with respect to $\phi$ and $\phi^{\prime}$.

Finally, implementing a two-level GPOF to the first part of (14) as explained in detail in [12] and adding the closed-form part of (14) to the resultant expression, CFGF representations in the spatial domain is obtained for magnetic sources.

\section{NUMERICAL RESULTS}

Numerical results in the form of mutual coupling between two apertures on the PEC layer of a dielectric coated circular PEC cylinder is considered to assess the accuracy and efficiency of the proposed CFGF representations. The apertures are modeled as tangential magnetic current modes, $M_{1_{u}}$ and $M_{2_{v}}$, using the surface equivalence theorem [13], and the developed CFGF representations are used to find the mutual admittance $Y_{12 u v}$ between them, and is given by

$$
Y_{12 u v}=\int_{S_{2}} M_{2_{v}}\left[\int_{S_{1}} M_{1_{u}} G_{u v} d s_{1}\right] d s_{2} .
$$

The results are then compared with those obtained from CST Microwave Studio (MWS) [16] simulations, where the apertures are modeled with small waveguides having dimensions $z_{a}$ and $l_{a}$ in the $z$ - and $l$-directions, respectively. Then, $M_{1_{u}}$ and $M_{2_{v}}$, used in the CFGF-based mutual admittance calculations, are obtained from the aperture electric field distributions of CST MWS simulations ( $\left.\mathbf{M}=\mathbf{E} \times \hat{a}_{\rho}\right)$, and a $u$-directed current mode is in the following form:

$$
M_{u}=\cos \left(\frac{\pi|u|}{u_{a}}\right) \text { if } u \in\left[\frac{-u_{a}}{2}, \frac{u_{a}}{2}\right], v \in\left[\frac{-v_{a}}{2}, \frac{v_{a}}{2}\right] .
$$

Fig. 2 shows the magnitude (in $\mathrm{dB}$ ) and the phase of mutual admittances $Y_{12_{z z}}, Y_{12_{\phi z}}$, and $Y_{12_{\phi \phi}}$ versus $\alpha$ for various $s$ values for a PEC cylinder with $a_{0}=3 \lambda_{0}, a_{1}=3.06 \lambda_{0}, \epsilon_{r}=3.25$. The dimensions of each aperture are selected to be $0.3 \lambda_{0}$ along the direction of the current and $0.1 \lambda_{0}$ along the other direction.

To illustrate the accuracy of the provided CFGF expressions along the axial line of the cylinder, the magnitude (in $\mathrm{dB}$ ) and phase of $Y_{12 z z}$ versus $s$ for $\alpha=90^{\circ}$ is depicted in Fig. 3 as an example. The cylinder and the apertures are kept the same. In all figures, the path parameters, the number of samples and the number of complex exponentials used in each leg of the deformed integration path (see [12]), that are used to generate the CFGF results, are similar to those reported in [12]. On the other hand, good agreement is obtained between the CFGF and the CST MWS results. The discrepancies in some plots are due to the difficulties in the convergence of CST MWS results. Finally, each mutual coupling versus $s$ result for a fixed $\alpha$ lasts approximately 1.5 hours with CST MWS on a remarkably powerful workstation. However, it takes approximately 25 seconds to generate the same result using the provided CFGF expressions on a regular PC using MATLAB. Regarding the efficiency of the provided CFGF expressions, it should be noted that the summation over $n$ is still the main bottleneck of the method, in particular for electrically very large cylinders. As the radius of the cylinder becomes large, convergence problems may exhibit (good results are obtained up to $a_{0}=5 \lambda_{0}$ ), and the method becomes less efficient compared to high frequency techniques.

\section{CONCLUSIONS}

CFGF expressions for tangential magnetic sources are developed to investigate aperture antennas on PEC cylinders coated with dielectric layers. The provided CFGF expressions are accurate in a significantly wider source-field point region compared to previously available representations. Furthermore, because of several techniques used in the course of derivations, mutual coupling results can be obtained accurately for both electrically small and relatively large cylinders, where the latter case has been usually accomplished using high-frequency based methods. The proposed CFGFs are not valid in the region where two magnetic sources touch/overlap with each other. Besides, 
in the course of obtaining CFGF expressions, the approximating functions represent spherical waves with complex distances. Hence, types of waves that are different in nature than spherical waves, such as surface waves are not represented properly. Consequently, the proposed CFGF representations are less accurate when the field point is electrically far away from the source location where surface waves start to dominate.

\section{REFERENCES}

[1] L. Josefsson and P. Persson, Conformal Array Antenna Theory and Design. Piscataway/Hoboken, NJ: IEEE Press/Wiley, 2006.

[2] P. Persson and R. G. Rojas, "High-frequency approximation for mutual coupling calculations between apertures on a perfect electric conductor circular cylinder covered with a dielectric layer: Nonparaxial region," Radio Sci., vol. 38, no. 4, Jul./Aug. 2003.

[3] Y. L. Chow, J. J. Yang, D. F. Fang, and G. E. Howard, "A closed-form spatial Green's function for the thick microstrip substrate," IEEE Trans. Microwave Theory Tech., vol. 39, pp. 588-592, Mar. 1991.

[4] G. Dural and M. I. Aksun, "Closed-form Green's functions for general sources and stratified media," IEEE Trans. Microwave Theory Tech., vol. 43, pp. 1545-1552, Jul. 1995.

[5] M. I. Aksun, "A robust approach for the derivation of closed-form Green's functions," IEEE Trans. Microwave Theory Tech., vol. 44, pp. 651-658, May 1996.

[6] C. Tokgöz, "Derivation of closed-form Green's functions for cylindrically stratified media," M.S. thesis, Dept. Elect. Electron. Eng., Middle East Technical Univ., Ankara, Turkey, 1997.

[7] C. Tokgöz and G. Dural, "Closed-form Green's functions for cylindrically stratified media," IEEE Trans. Microwave Theory Tech., vol. 48, pp. 40-49, Jan. 2000.

[8] J. Sun, C.-F. Wang, L.-W. Li, and M.-S. Leong, "A complete set of spatial-domain dyadic Green's function components for cylindrically stratified media in fast computational form," J. Electromagn. Waves Appl., vol. 16, no. 11, pp. 1491-1509, 2002.

[9] R. C. Acar and G. Dural, "Comments on a complete set of spatialdomain dyadic Green's function components for cylindrically stratified media in fast computational form," J. Electromagn. Waves Appl., vol. 18, no. 10, pp. 1389-1394, 2004.

[10] J. Sun, C.-F. Wang, L.-W. Li, and M.-S. Leong, "Reply to comments on a complete set of spatial-domain dyadic Green's function components for cylindrically stratified media in fast computational form," $J$. Electromagn. Waves Appl., vol. 18, no. 10, pp. 1395-1398, 2004.

[11] J. Sun, C.-F. Wang, L.-W. Li, and M.-S. Leong, "Further improvement for fast computation of mixed potential Green's functions for cylindrically stratified media," IEEE Trans. Antennas Propag., vol. 52, pp. 3026-3036, Nov. 2004

[12] S. Karan, V. B. Erturk, and A. Altintas, "Closed-form Green's function representations in cylindrically stratified media for method of moments applications," IEEE Trans. Antennas Propag., vol. 57, pp. 1158-1168, Apr. 2009.

[13] C. A. Balanis, Advanced Engineering Electromagnetics. New York: Wiley, 1989.

[14] Y. Hua and T. K. Sarkar, "Generalized pencil-of-function method for extracting poles of an EM system from its transient response," IEEE Trans. Antennas Propag., vol. 37, pp. 229-234, Feb. 1989.

[15] M. Abramowitz and I. A. Stegun, Handbook of Mathematical Functions With Formulas, Graphs, and Mathematical Tables. Mineola, NY: Dover, 1970.

[16] CST MWS, SP1 [DVD-ROM]. Darmstadt, Germany, 2011.

\section{Multiwall Carbon Nanotubes at RF-THz Frequencies: Scattering, Shielding, Effective Conductivity, and Power Dissipation}

Jay A. Berres and George W. Hanson

Abstract-Isolated, infinitely long multiwall carbon nanotubes (MWCNTs) interacting with electromagnetic waves in the microwave and far-infrared frequency regime are analyzed using a semi-classical approach. An expression for the bulk effective conductivity of MWCNTs is obtained, valid up to $\mathrm{THz}$ frequencies. The influence of the number of tube walls, the radius of the outermost tube wall, and the presence of a gold core on scattering and shielding is analyzed. Comparisons between metallic MWCNTs, metallic single wall carbon nanotubes (SWCNTs), and metal nanowires are provided.

Index Terms-Carbon nanotube, electromagnetic theory, nanotechnology.

\section{INTRODUCTION}

Carbon nanotubes (CNTs) continue to be at the forefront of research today, since their physical properties make them promising candidates for nanoscale applications. They can form naturally into two types, single wall carbon nanotubes (SWCNTs) and multiwall carbon nanotubes (MWCNTs). A MWCNT consists of multiple co-centric SWCNTs, where the distance between each tube wall is approximately $0.34 \mathrm{~nm}$, which is the distance between interatomic layers of graphite (i.e., graphene sheets) [1]. The number of tube walls for a MWCNT can vary anywhere from 2 to several hundred. Typically the length of CNTs can be from the nanometer scale up to centimeters, and in the case of SWCNTs, their cross-sectional radius varies within the range of approximately 0.3 to $2-5 \mathrm{~nm}$. For MWCNTs, their overall cross-sectional radius varies within the range of approximately 1 to $100 \mathrm{~nm}$. The electromagnetic response of CNTs, and their corresponding applications as antennas, interconnects, and thermal contrast agents, are being investigated [2]-[15]. Of the papers that analyze the electromagnetic response of CNTs, the majority of these papers focus on SWCNTs, although [8] and [12] consider MWCNTs, [9] and [11] consider nanotube bundles, and [13] and [14] consider nanotube sheets. From the emerging literature it is becoming clear that for far-infrared applications, individual SWCNTs have losses that are too large (associated with their extremely small radius) to serve as antennas or interconnects. However, bundles of SWCNTs, and individual or bundles of MWCNTs, may be good candidates for antenna and interconnect applications. Furthermore, planar sheets of nanotubes fabricated as conformal patch antennas have shown excellent properties [13], [14].

In this work, the electromagnetic response of an isolated, infinitely long MWCNT is analyzed in the microwave and far-infrared frequency regime using a semi-classical approach. The influence of the number of tube walls, the radius of the outermost tube wall, and the presence of a gold core on scattering and shielding characteristics is analyzed. Comparisons between metallic MWCNTs, metallic SWCNTs, and metal

Manuscript received May 17, 2010; revised November 18, 2010; accepted January 15,2011 . Date of publication June 09,2011 ; date of current version August 03, 2011.

The authors are with the Department of Electrical Engineering, University of Wisconsin-Milwaukee, Milwaukee, WI 53211 USA (e-mail: jaberres@gmail. com; george@uwm.edu).

Digital Object Identifier 10.1109/TAP.2011.2158951 